\title{
ENSAYO DE PROPAGACION DE VARIEDADES DE VID EN SELVA ALTA
}

\author{
Castañeda Ruíz Carlos ( $\left.{ }^{*}\right)$ \\ Garayar M eléndez Héctor $\left({ }^{* *}\right)$
}

\section{RESUMEN}

El cultivo de la variedad Borgoña negra (Isabella) es de uso corriente en el departamento de San Martín, ubicado en la selva alta del país. Para investigar si otros cultivares de la costa central pueden adaptarse a las condiciones climáticas de selva alta, se está ensayando el cultivo de catorce variedades procedentes del departamento de Ica.

Los trabajos se realizaron durante los años 1988-89 en la Estación Experimental El Porvenir, localizada en el distrito de Juan Guerra, provincia y departamento de San Martín, a 356 m.s.m.m. La temperatura media anual osciló alrededor de 26.5 ㅇ $\mathrm{C}$ y las precipitaciones bordearon los 1,000 mm. En el mismo período.

Durante los años 1988 y 1989 se examinó la propagación, plantando estacas sin injertar en bolsas de polietileno negro, de $15 \times 30 \mathrm{~cm}$., conteniendo 2 kilos de sustrato. De este modo, se evitó las dificultades que en el plantado directo y en la formación de almácigos en el suelo originan el exhuberante crecimiento de la maleza o la desecación en los períodos de escasa pluviosidad.

En 1988, los almácigos se formaron con estacas de no menos de $6 \mathrm{~mm}$. L legando a tener hasta más de $12 \mathrm{~mm}$. De diámetro, dejando 1 a 4 yemas al aire. EI prendimiento promedio alcanzó el $41.8 \%$, variando entre el $22 \%$ exhibido por la variedad "Albilla' y el 76.2\% que correspondió a la "Cardinal" (Cuadro №1). Se halló que el prendimiento fue directamente proporcional al grosor de las estacas e inversamente al número de yemas vistas.

En el segundo año, se emplearon estacas entre 7 y10 mm. De diámetro con sólo 1 a 2 yemas a la vista, sin variarlos factores de vivero, riego, sustrato, bolsas y personal. Los elementos climáticos se manifestaron similares a los del año precedente. Sin embargo, el nivel de prendimiento en el almácigo ascendió en promedio a $81.9 \%$, distribuyéndose entre el $43.3 \%$ de la variedad Torontel" y el $100 \%$ de "Negra corriente".

El método de propagación utilizado facilitó el control de la humedad del sustrato, el manejo fitosanitario de los plantones, su transporte y el plantado en el viñedo.

\footnotetext{
Director del Centro Regional de Investigación - IIA P - San M artín

** Ingeniero A grónomo, Investigador del IMAA, Estación Experimental “Los Pobres" - Ica
} 
El objetivo de la investigación fue establecer una tecnología apropiada para la propagación de vides costeñas en las condiciones climáticas y agronómicas típicas de este departamento $y$, por extensión, de la selva alta norte. Como el material genético proviene de la poda que se cumple una vez al año en Ica, los ensayos sólo pudieron verificarse con esa periodicidad.

\section{REVISION DE LA LITERATURA}

Winkler (6), señaló en 1984 que, en viticultura, casi todas las variedades dedicadas para frutificación para patrones enraizados se propagan por estacas. Estas, generalmente, se cultivan en un vivero durante un año para producir los barbados; pero, en algunas ocasiones, se plantan directamente en el viñedo estacas sin enraizamiento.

El mismo autor, op. cit., indica que los sarmientos más deseables para estacas son de tamaño medio y con entrenudos de longitud moderada. Losentre nudos muy cortos son signos casi siempre de enfermedad o malas condiciones de crecimiento. Con las variedades de Vitis vinifera, las estacas tienen comúnmente diámetros de 8.5 a 12.7 $\mathrm{mm}$., las menores de $6.4 \mathrm{~mm}$. En su extremidad inferior deben rechazarse. L a longitud de las estacas para plantaciones en vivero varía de 30 a $45 \mathrm{~cm}$., prefiriéndose de 30 a 40 para estacas francas y de 40 a 45 para pies de injerto. También dice que la plantación de estacas sin enraizar es siempre peligrosa, resultando usualmente en malas plantaciones. L as plantas de estacas sin enraizar (salvo de que el plantío se maneje con tanto cuidado como en el vivero) no darán un promedio de más del $85 \%$ y con frecuencia será menor del $60 \%$. El replante se hace necesario, debido a estacas sin enraizar, pero es caro y resultará en un viñedo desigual. L as vides replantadas rara vez alcanzan 0 se igualan con aquellas de la plantación original. Por todo eso, no debe promoverse mucho el empleo de estacas para plantar directamente un viñedo. Se agrega que el método puede resultar ventajoso por su bajo costo inicial, si se evita la contaminación; además, el terreno debe estar libre de malezas, de filoxera y/o nemátodos, y las demás condiciones de prendimiento deben ser favorables. Tratándose de estacas sin injertar, Rodríguez y Ruesta (5), citaron en 1982 que pueden plantarse en campo definitivo o en almácigo. La primera forma elimina el retraso y el gasto que significa el trasplante pero expone bruscamente el material a un medio poco favorable. En el caso de almácigos, recomendaron un distanciamiento de $10 \mathrm{~cm}$. entre estacas y $1 \mathrm{~m}$. Entre líneas, así como enterrar las estacas de $30 \mathrm{~cm}$., dejando 1 ó 2 yemas al aire y proporcionándoles riegos ligeros y frecuentes hasta el prendimiento En la Guía Técnica del Viticultor (4)1984, se puntualiza que la calidad de planta que se obtiene de sarmientos, está influenciada por las características de la planta madre; por lo tanto, se recomienda marcar las plantas donadoras de sarmientos. Estos deben seleccionarse de plantas productivas, con buen vigor, sanas, y no deben utilizarse aquellas cuyo follaje haya mostrado durante su ciclo de crecimiento alguna anormalidad. Se debe utilizar únicamente madera bien agostada 0 madura y, de ese modo, se obtienen las siguientes ventajas: reducir el porcentaje de plantas enfermas, establecer material con mayores posibilidades de producción, evitar la mezcla de variedades y aumentar el porcentaje de brotación en el vivero. 
A simismo, en otro acápite de la citada obra, se sostiene que debe evitarse defoliaciones otoñales, ya que estas provocan que la madera no acumule las reservas alimenticias necesarias y que no madure adecuadamente, lo cual reduce considerablemente la brotación y desarrollo de los sarmientos en el vivero.

\section{MATERIALES Y METODOS}

Las investigaciones que se reportan se realizaron entre agosto del 1988 y octubre de 1989, en la Estación Experimental "EI Porvenir" del INIAA, ubicada en el distrito J uan Guerra, provincia y departamento de San M artín, a 06 34' de latitud sur, $76020^{\prime}$ de longitud oeste, y $356 \mathrm{msnm}$. Durante el período citado se verificó un promedio de temperaturas máxima de 32.28 grados centígrados y mínimas de 20.4 . En el mes de setiembre de 1989, la temperatura máxima alcanzó el mayor registro, con una media de $34.2^{\circ} \mathrm{C}$, mientras que en julio del mismo año se había observado las menores mínimas, con un media mensual de $18.4^{\circ}$ C. (Figura 1). La precipitación anual bordeó Ios 1,050 mm., distinguiéndose períodos más lluviosos de febrero a mayo y de octubre a noviembre. (Figura 2).

Los sarmientos utilizados proceden de la Estación Experimental Los Pobres" del IN IAA-Ica, y tanto en 1988 como en 1989 fueron cortados a $60 \mathrm{~cm}$., desinfectados contra filoxera, embalados en paquetes de 200 y trasladados a San M artín, en períodos de 3 días, en ambas ocasiones.

En 1988, sólo se consiguió en Ica sarmientos de las once variedades siguientes: Italia blanca, Italia rosada, Cereza, Negra corriente, Albilla, Torontel, Alphonse L avallée (Rivier), M oscatel, Quebranta, I talia negra y Cardinal.

Se utilizó como testigo la variedad de cultivo tradicional en San Martín, Borgoña negra (Isabella), y los sarmientos se consiguieron en el distrito de San A ntonio de Cumbaza.

En 1989 se obtuvo en Ica sarmientos de las dieciocho variedades que se indican a continuación: Italia blanca, Italia rosada, Cereza, N egra corriente, Albilla, Torontel, Alphonse Lavalleé (Rivier), M oscatel, Quebranta, Italia negra, Cardinal, Thompson seedless, Chenin, Cabernet sauvignon, Riesling, Barbera, Grenache, $\mathrm{M}$ al bec.

L a identificación de las variedades corresponde a los registros del IN IA A - Ica.

Para los ensayos de prendimiento de las estacas se estableció tanto en 1988 como en 1989 almácigos en la Estación Experimental "El Porvenir", en un vivero sin techado, donde se obtuvo una mediana protección contra la fuerte iluminación solar, colocando hojas de palmera "Shapaja" a modo de techo.

En 1988 se prepararon estacas de $35 \mathrm{~cm}$. yen 1989 de 25 a 30, descartándose los sarmientos defectuosos o muy delgados. Las estacas se plantaron en bolsas de polietileno de $30 \mathrm{~cm}$. de largo, por 15 de ancho, de color negro y con perforaciones de $0.5 \mathrm{~cm}$. de diámetro. Cada bolsa fue llenada con $2 \mathrm{~kg}$. De tierra, que alcanzaba una al tura de $22 \mathrm{~cm}$. L as características del sustrato empleado, fueron las siguientes: 
$\begin{array}{ll}\text { A rena } & 58 \% \\ \text { Limo } & 20 \% \\ \text { A rcilla } & 22 \%\end{array}$

Textura Franco areno arcilloso

$P$

$\mathrm{K}$

$\mathrm{pH}$

M.O (materia orgánica)

$\mathrm{K}$

$\mathrm{Ca}+\mathrm{Mg}$.
IOppm.

$156 \mathrm{ppm}$.

En el ensayo de 1988, las estacas fueron clasificadas como delgadas (hasta 6 $\mathrm{mm}$. de diámetro), medianas (de 7 a $10 \mathrm{~mm}$.) y gruesas (más de $10 \mathrm{~mm}$.), y al plantarse se les dejó de 1 a 4 yemas al aire. En 1989, el almácigo se hizo sólo con estacas medianas, normalmente las más abundantes en Ica, y se les dejó 1 a 2 yemas al aire. En los dos casos, luego del plantado, la tierra de las bolsas previamente humedecida se regó a saturación, repitiéndose dicha práctica cada 2 días cuando no llovía. El almácigo se conformó con grupos de 150 bolsas, reunidas según variedades. Entre grupos se observó una separación de $50 \mathrm{~cm}$. En 1988 se trabajó con 300 estacas por variedad, mientras que en 1989 sólo con 150. Las labores de plantado de estacas se realizaron durante 12 días en 1988 y 15 días en 1989, con lo cual, añadido el período de corte y transporte totalizó 15 y 18 días, respectivamente, para todo el proceso de establecimiento de los almácigos.

En condiciones normales, los ayudantes de campo llenaron 200 bolsas diarias cada uno; la preparación de las estacas y el plantado lo realizaron los autores, a razón de 300 diarias cada uno. El manejo y toma de datos del almácigo ocupó cuatro horashombres diarios.

La fase prendimiento duró 64 días en 1988 y 56 en 1989 y abarcó el lapso entre el corte de los sarmientos y la evaluación. Durante ese período se observó diariamente el comportamiento de las estacas pero no se realizó tratamiento fitosanitario, a fin de examinar la respuesta natural de las variedades a los factores adversos.

La evaluación de la fase prendimiento comprendió el porcentaje de estacas prendidas por variedad, considerando grosor y número de yemas, así como el vigor de los brotes. Se entiende por estacas prendidas aquellas con más de 7 horas, al haberse comprobado que en ese estadio de desarrollo ya poseen raicillas de cinco 0 más centímetros y que al plantarse en el suelo continúan su crecimiento.

\section{RESULTADOS Y DISCUSION}

Las Figuras 1 y 2 ilustran sobre las condiciones climáticas en las que se desarrollaron los ensayos, resaltando la temperatura que, según Winkler (6), es el factor que más modifica el comportamiento de la vid. En Ica se realiza el proceso de propagación en el sistema de estratificación, en la misma época pero en período más largo, con temperaturas máximas y mínimas de 26.5 y $112^{\circ}$ C, respectivamente, (Promedio agosto- 
octubre de los años (1986 -1988) y con resultados semejantes. Resulta visible que el apreciable nivel y la escasa variación diaria de temperatura experimentados en la Estación El Porvenir, no afectaron el prendimiento de las estacas, sino más bien lo aceleraron. Al vincular el resultado de los ensayos con la importante proporción de arena del sustrato utilizado para los almácigos, se corrobora la idea generalizada de emplear tierra arenosa para un mejor prendimiento de las estacas. Hace falta ensayar la propagación con otros tipos de sustrato para tener una idea más concluyente. En los Cuadros № 1 al 5, se aprecian las disimilitudes en el prendimiento registradas para las mismas variedades en los años 1988 y 1989. Si consideramos que las fechas de corte de los sarmientos han sido idénticas para las variedades examinadas, tanto en 1988 como en 1989, y que los períodos desde el corte o el plantado hasta la finalización del brotamiento no guardan relación con el prendimiento, en ninguno de los años, se puede asumir que el considerable aumento en el grado de prendimiento entre 1988 (41.75\%) y 1989 (81.9\%), se debe a la utilización en este último año, de estacas de grosor mediano, plantadas solo con 1 y 2 yemas al aire, confirmando lo expresado en los Cuadros N 26 y 7. Al plantar estacas con 1 ó 2 yemas vistas se reduce el tamaño de esas a un promedio de $28 \mathrm{~cm}$.; así, se obtiene de un sarmiento mayor cantidad, se reduce el costo de cada una y se rebaja el gasto en flete. Como ventaja adicional, considerase el mayor porcentaje en el prendimento cuando se deja 162 yemas al aire. En los Cuadros $\mathrm{N}$ ㅇ 2 y 3 , se presenta la evolución durante los años 1988 y 89 del brotamiento que experimentaron las variedades, vinculando su aumento porcentual con el lapso transcurrido, desde el corte. Es de notar que en el año 1989 la evolución fue más rápida que en el precedente, pese a que en éste, el brotamiento se inició dos días antes en promedio. En 1988, el brotamiento se desarrolló en todos los cultivares con mayor intensidad hasta los 34 días después del corte; entonces, se produjo un brusco y enérgico marchitamiento, que redujo las estacas brotadas de 1,412 a 1,150, es decir, a un $81.44 \%$. Este colapso afecté en forma diferente a todas las variedades, excepto a I talia blanca. No se hallé explicación al fenómeno, más aún cuando las condiciones climáticas, especialmente la temperatura, tuvieron un comportamiento regular en los días anteriores. Las estacas continuaron brotando hasta los 58 días, aunque en el Cuadro № 2 sólo se indica el período transcurrido en general hasta el colapso, el brotamiento posterior no llegó a superar el nivel de ese entonces, en la mayoría de los casos.

En 1989, el brotamiento se alargó después del corte hasta los 50-55 días para las variedades Alphonse Lavallée, Italia rosada, Italia blanca y Cereza, mientras que Negra corriente alcanzó el $100 \%$ a los 41 días solamente. Según lo anotado en los Cuadros №1, 2 y 3 , se observa que durante el ensayo, las estacas empezaron a brotar, en promedio, a los 18 días posteriores al corte y terminaron luego de 34 y 32 días, en 1988 y 1989, respectivamente. No se ha comprobado una correlación entre el período corte-fin del brotamiento y el porcentaje de prendimiento, en ninguno de los años (1988 $r=0.46 ; 1989, r=0.41)$. Tampoco se ha confirmado correlación entre los períodos de remojo (previo al plantado en almácigo) y el prendimiento (1988 $r=0.36 ; 1989 r=$ 
0.28), ni entre los períodos de plantado hasta el inicio del brote y el prendimiento (1989 $r=0.61 ; 1989 r=0.06$ ). (Cuadros $N \cong 4$ y 5 ).

En 1988, el brotamiento máximo difirió del prendimiento en amplia medida 


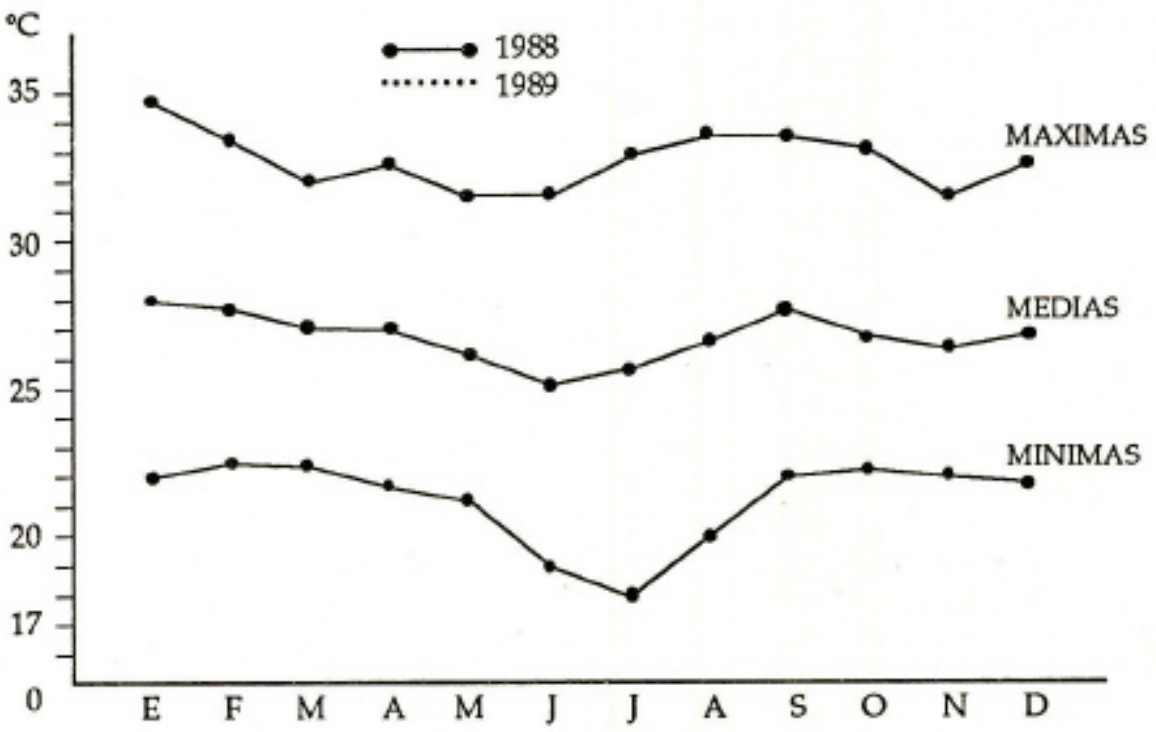

FIGURA 1: Promedio mensual de temperaturas en la Estación Experimental El Porvenir en los años 1988 y 1989.

Fuente: Servicio Nacional de Meteorología e Hidrología: SENAMHI

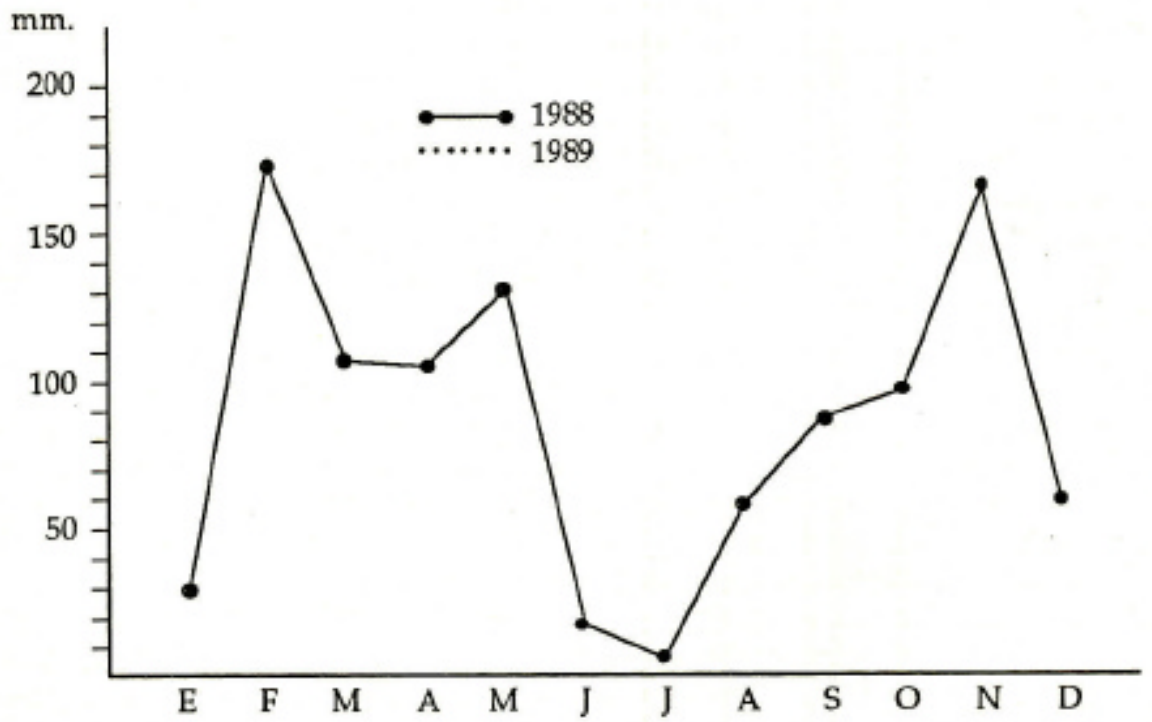

FIGURA 2. Registro pluviométrico mensual en la Estación Experimental El Porvenir, en lo: años 1988 y 1989.

Fuente: SENAMHI 


\begin{tabular}{|c|c|c|c|c|c|c|c|c|c|c|c|c|c|c|c|}
\hline & & 兽惫 & $\infty$ & $\stackrel{\infty}{\infty}$ & $\tilde{\alpha}$ & ळे & ‡ & $\underset{\infty}{\infty}$ & \& & ఖ & $b$ & $\tilde{\infty}$ & $R$ & 8 & ' \\
\hline$\underset{\sum}{E}$ & & 焉 & $\infty$ & 오 & $\begin{array}{l}\text { ?ु } \\
\text { cै }\end{array}$ & $\stackrel{\infty}{\vec{d}}$ & 过 & ชู & व̊ำ & 동 & $\vec{g}$ & 守 & กั & 离 & 욤 \\
\hline$\frac{\vec{z}}{\frac{a}{a}}$ & & 占总 & $\mathscr{\infty}$ & $\frac{}{ㅁ}$ & $\begin{array}{l}\stackrel{0}{7} \\
\dot{8}\end{array}$ & $\frac{0}{8}$ & $\stackrel{ }{ }$ & $\begin{array}{l}\stackrel{ }{ } \\
\check{8}\end{array}$ & $\begin{array}{l}8 \\
\text { ñ }\end{array}$ & $\frac{0}{8}$ & $\begin{array}{l}\stackrel{ }{8} \\
8\end{array}$ & $\begin{array}{l}\stackrel{ }{8} \\
\dot{8}\end{array}$ & $\frac{0}{9}$ & $\frac{0}{\circ}$ & ' \\
\hline$\frac{\sqrt{0}}{2}$ & & 氯总 & gू & $\begin{array}{l}\stackrel{0}{\ominus} \\
\dot{0}\end{array}$ & $\stackrel{0}{0}$ & $\begin{array}{l}\stackrel{\theta}{1} \\
\stackrel{1}{0}\end{array}$ & $\stackrel{0}{\circ}$ & $\begin{array}{l}\stackrel{0}{7} \\
\stackrel{2}{r}\end{array}$ & $\frac{0}{5}$ & $\begin{array}{l}\stackrel{0}{0} \\
\stackrel{1}{a}\end{array}$ & $\begin{array}{l}\stackrel{0}{\beth} \\
\stackrel{ }{ }\end{array}$ & $\frac{}{\stackrel{\text { ํ }}{\circ}}$ & $\stackrel{ }{\stackrel{0}{*}}$ & $\stackrel{ }{ }$ & $\begin{array}{l}\exists \\
\dot{8}\end{array}$ \\
\hline $\begin{array}{l}7 \\
\text { 參 } \\
0\end{array}$ & & .2. ․ㅗㄹ & \&ి & $\begin{array}{l}8 \\
\dot{8} \\
\dot{8}\end{array}$ & $\begin{array}{l}8 \\
8\end{array}$ & $\frac{g}{8}$ & $\begin{array}{l}8 \\
1 \\
1\end{array}$ & $\begin{array}{l}8 \\
1 \\
8\end{array}$ & $\begin{array}{l}8 \\
\dot{0}\end{array}$ & $\begin{array}{l}8 \\
\dot{0} \\
8\end{array}$ & $\begin{array}{l}8 \\
\dot{8} \\
b\end{array}$ & $\begin{array}{l}8 \\
1 \\
b\end{array}$ & $\begin{array}{l}8 \\
0 \\
8\end{array}$ & $\begin{array}{l}\text { g } \\
1 \\
8\end{array}$ & ' \\
\hline 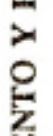 & $\begin{array}{l}9 \\
\text { న్ }\end{array}$ & 包嶨 & $\infty$ & $\begin{array}{l}8 \\
=\end{array}$ & $\begin{array}{l}8 \\
1 \\
8\end{array}$ & $\begin{array}{l}g \\
\dot{8} \\
0 \\
0\end{array}$ & $\begin{array}{l}g \\
1 \\
b\end{array}$ & $\begin{array}{l}8 \\
1 \\
8\end{array}$ & $\begin{array}{l}8 \\
1 \\
0 \\
0\end{array}$ & $\begin{array}{l}g \\
\dot{g} \\
g\end{array}$ & $\begin{array}{l}8 \\
=\end{array}$ & $\begin{array}{l}8 \\
1 \\
=\end{array}$ & $\begin{array}{l}8 \\
\dot{0} \\
\stackrel{-}{0}\end{array}$ & $\begin{array}{l}8 \\
\dot{1} \\
\stackrel{-}{1}\end{array}$ & $\begin{array}{l}\text { g } \\
\dot{0} \\
\stackrel{\sim}{0}\end{array}$ \\
\hline$\frac{3}{6}$ & $\begin{array}{l}\infty \\
\infty \\
\infty\end{array}$ & 윰 & ఏి & $\begin{array}{l}\text { g̊ } \\
\dot{1} \\
\text { ते }\end{array}$ & $\begin{array}{l}0 \\
0 \\
\dot{d} \\
\dot{d}\end{array}$ & $\begin{array}{l}\text { \& } \\
\stackrel{1}{d}\end{array}$ & $\begin{array}{l}\infty \\
\ddot{0}\end{array}$ & $\begin{array}{l}\infty \\
0 \\
\dot{~}\end{array}$ & 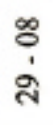 & $\begin{array}{l}\infty \\
\dot{0} \\
\ddot{d}\end{array}$ & $\begin{array}{l}\infty \\
0 \\
\dot{d}\end{array}$ & $\begin{array}{l}\infty \\
0 \\
\dot{2}\end{array}$ & $\begin{array}{l}\stackrel{\infty}{0} \\
\dot{1} \\
\text { ปี }\end{array}$ & $\begin{array}{l}\text { O } \\
\text { 영 }\end{array}$ & , \\
\hline రీ & r & 配 & $\stackrel{\infty}{\infty}$ & $\begin{array}{l}\infty \\
0 \\
\dot{0} \\
\text { ते }\end{array}$ & $\begin{array}{l}\infty \\
0 \\
1 \\
\text { वे }\end{array}$ & $\frac{\infty}{\dot{0}}$ & $\begin{array}{c}\infty \\
0 \\
\dot{1} \\
m\end{array}$ & $\begin{array}{l}8 \\
1 \\
5\end{array}$ & $\begin{array}{l}8 \\
\dot{0} \\
\dot{0}\end{array}$ & $\begin{array}{l}8 \\
0 \\
8\end{array}$ & $\begin{array}{l}8 \\
\dot{8} \\
8\end{array}$ & $\begin{array}{l}8 \\
\dot{8} \\
\dot{0}\end{array}$ & $\begin{array}{l}8 \\
1 \\
5\end{array}$ & $\begin{array}{l}8 \\
0 \\
0\end{array}$ & $\begin{array}{l}\text { gे } \\
\dot{1} \\
\text { 언 }\end{array}$ \\
\hline Z & & & வ & $\begin{array}{l}\infty \\
\dot{1} \\
\dot{2}\end{array}$ & $\begin{array}{l}\infty \\
\dot{0} \\
2\end{array}$ & $\begin{array}{l}\infty \\
\vdots \\
\vdots\end{array}$ & $\begin{array}{l}\infty \\
0 \\
\dot{2}\end{array}$ & $\begin{array}{l}\infty \\
0 \\
\dot{a} \\
\sim\end{array}$ & $\begin{array}{l}\infty \\
0 \\
n\end{array}$ & $\begin{array}{l}\infty \\
0 \\
1 \\
2\end{array}$ & $\begin{array}{l}\infty \\
0 \\
\dot{2} \\
\sim\end{array}$ & $\begin{array}{l}9 \\
0 \\
1 \\
2\end{array}$ & $\begin{array}{l}\infty \\
0 \\
1 \\
n\end{array}$ & $\begin{array}{l}\infty \\
0 \\
2 \\
2\end{array}$ & 1 \\
\hline 응 & & 8 & $\infty$ & $\begin{array}{l}\infty \\
\dot{8} \\
\text { ते }\end{array}$ & \begin{tabular}{l}
$\infty$ \\
0 \\
\cline { 1 - 1 }
\end{tabular} & $\begin{array}{l}\infty \\
\dot{8}\end{array}$ & $\begin{array}{l}\text { o } \\
\text { নं }\end{array}$ & $\begin{array}{l}\mathscr{g} \\
\dot{2}\end{array}$ & $\begin{array}{l}\mathscr{8} \\
\ddot{1}\end{array}$ & $\begin{array}{l}\infty \\
\dot{0} \\
\dot{1}\end{array}$ & $\begin{array}{l}\text { g } \\
\text { ภ่ }\end{array}$ & $\begin{array}{l}\infty \\
\dot{8} \\
\end{array}$ & $\begin{array}{l}\infty \\
\dot{0} \\
\dot{2}\end{array}$ & $\begin{array}{l}\infty \\
\dot{0} \\
\text { वें }\end{array}$ & $\begin{array}{l}8 \\
\dot{0} \\
-1\end{array}$ \\
\hline 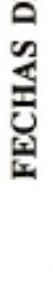 & & $\begin{array}{l}\frac{9}{3} \\
\text { 量 } \\
\frac{1}{3}\end{array}$ & & 总 & $\begin{array}{l}\text { 总 } \\
\text { 足 } \\
\text { 总 } \\
\text { 롤 }\end{array}$ & 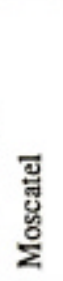 & 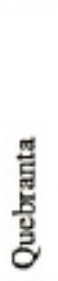 & 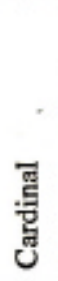 & 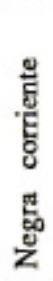 & 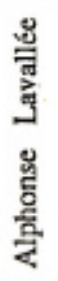 & 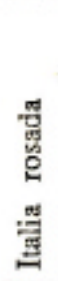 & $\begin{array}{l}\text { 总 } \\
\text { 总 } \\
\text { 莺 }\end{array}$ & 迩 & $\begin{array}{l}\bar{\Xi} \\
\text { है } \\
\text { E }\end{array}$ & 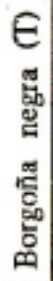 \\
\hline
\end{tabular}




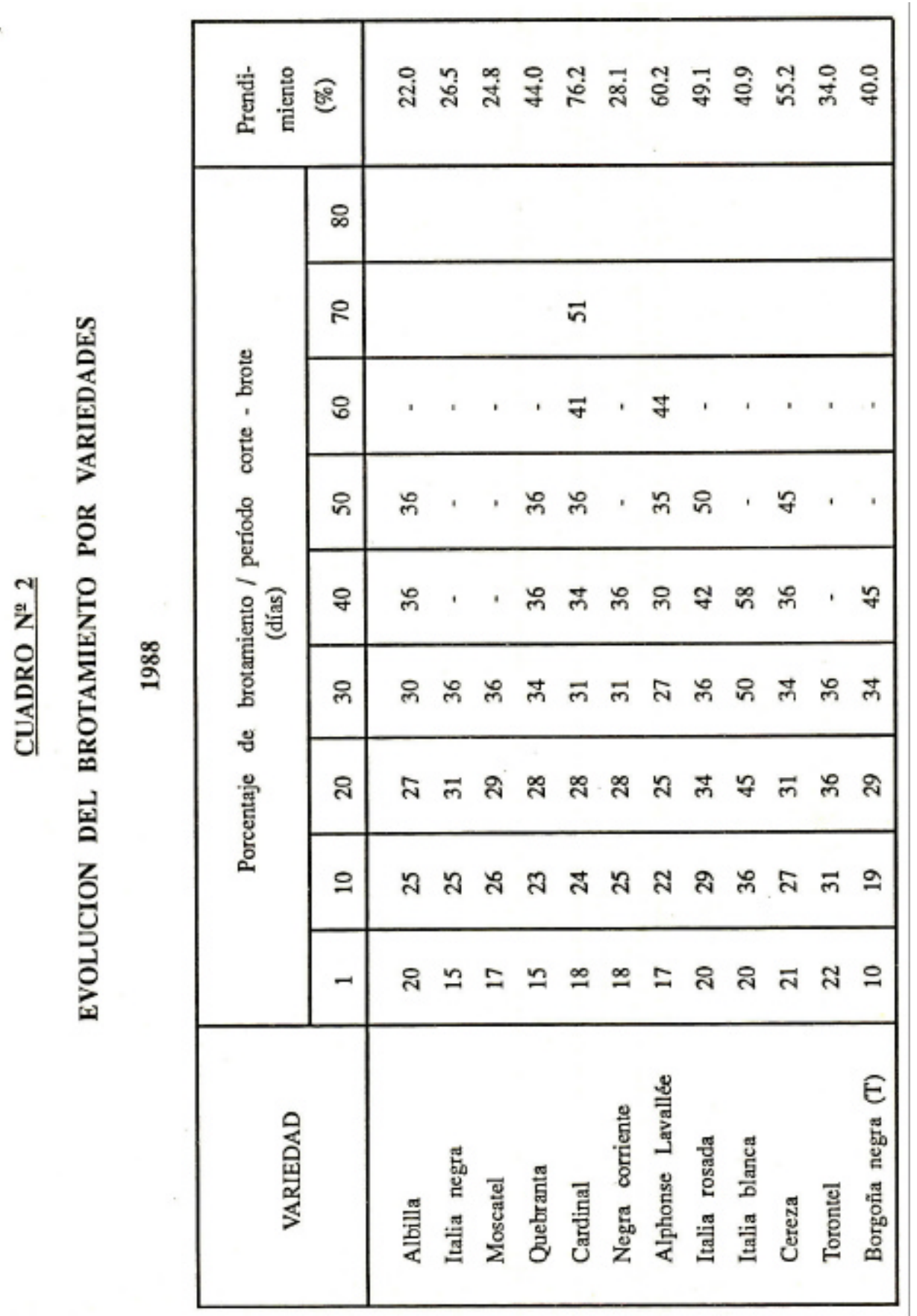




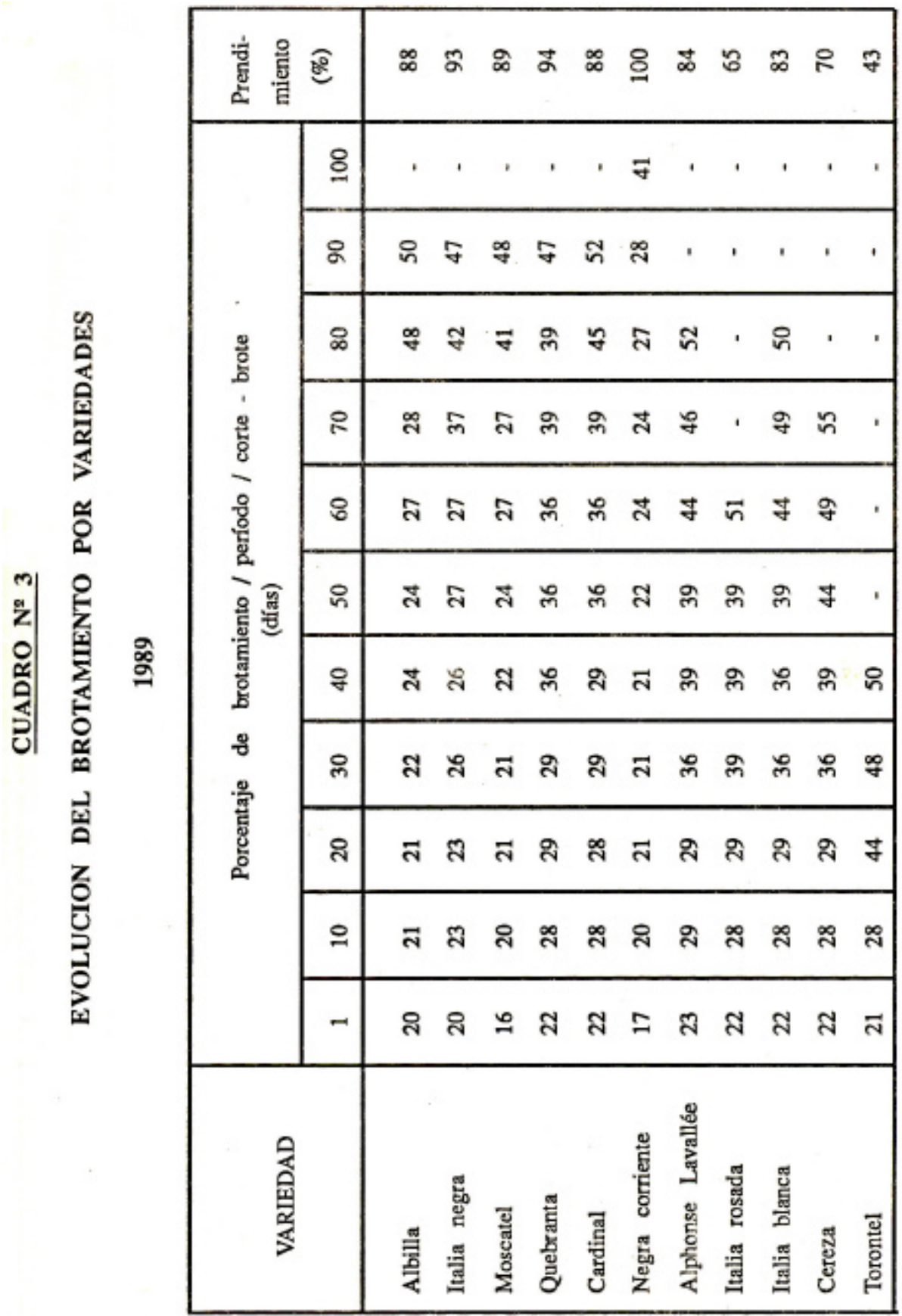




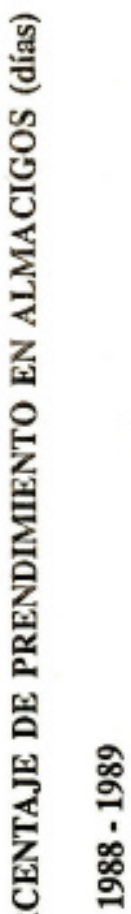

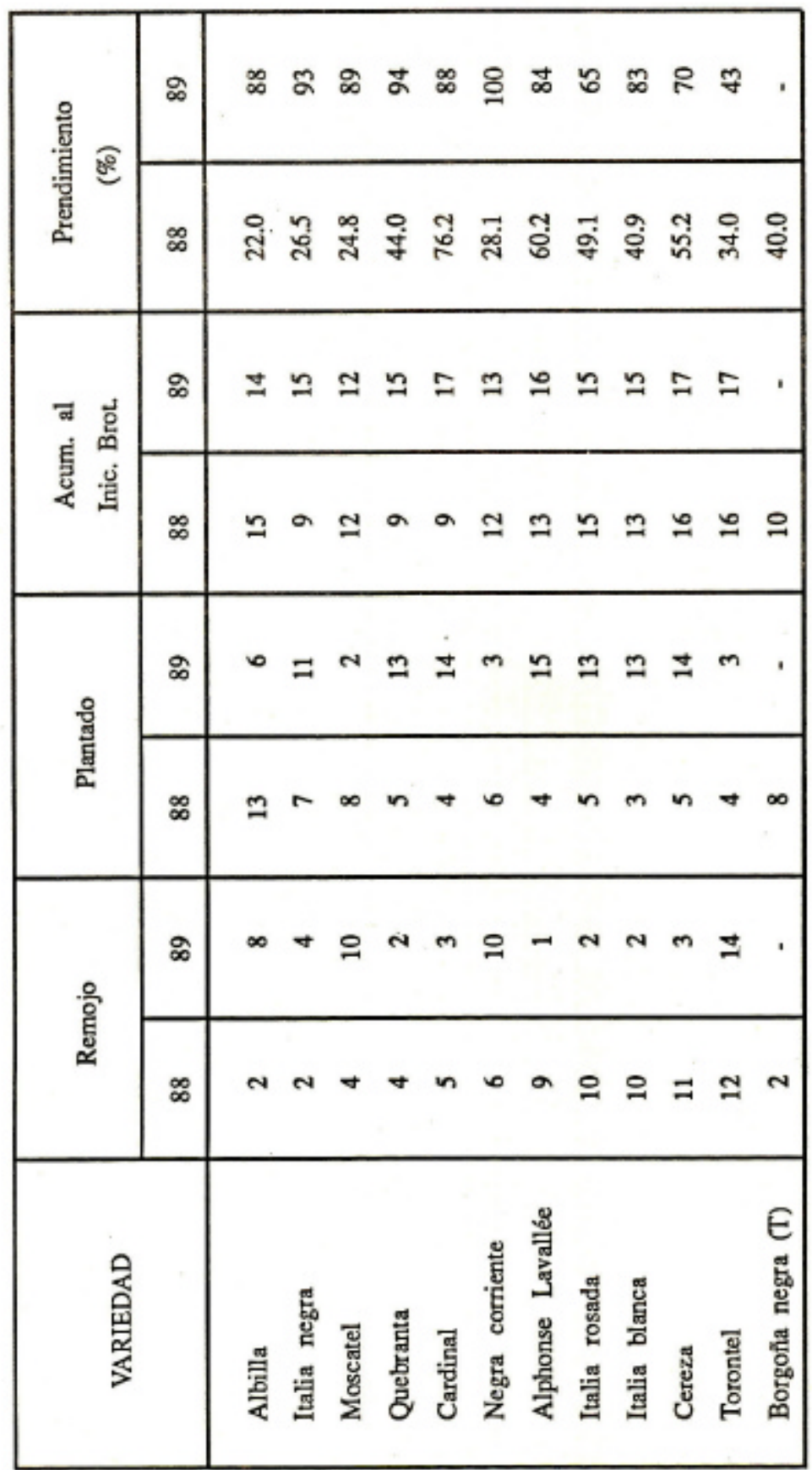




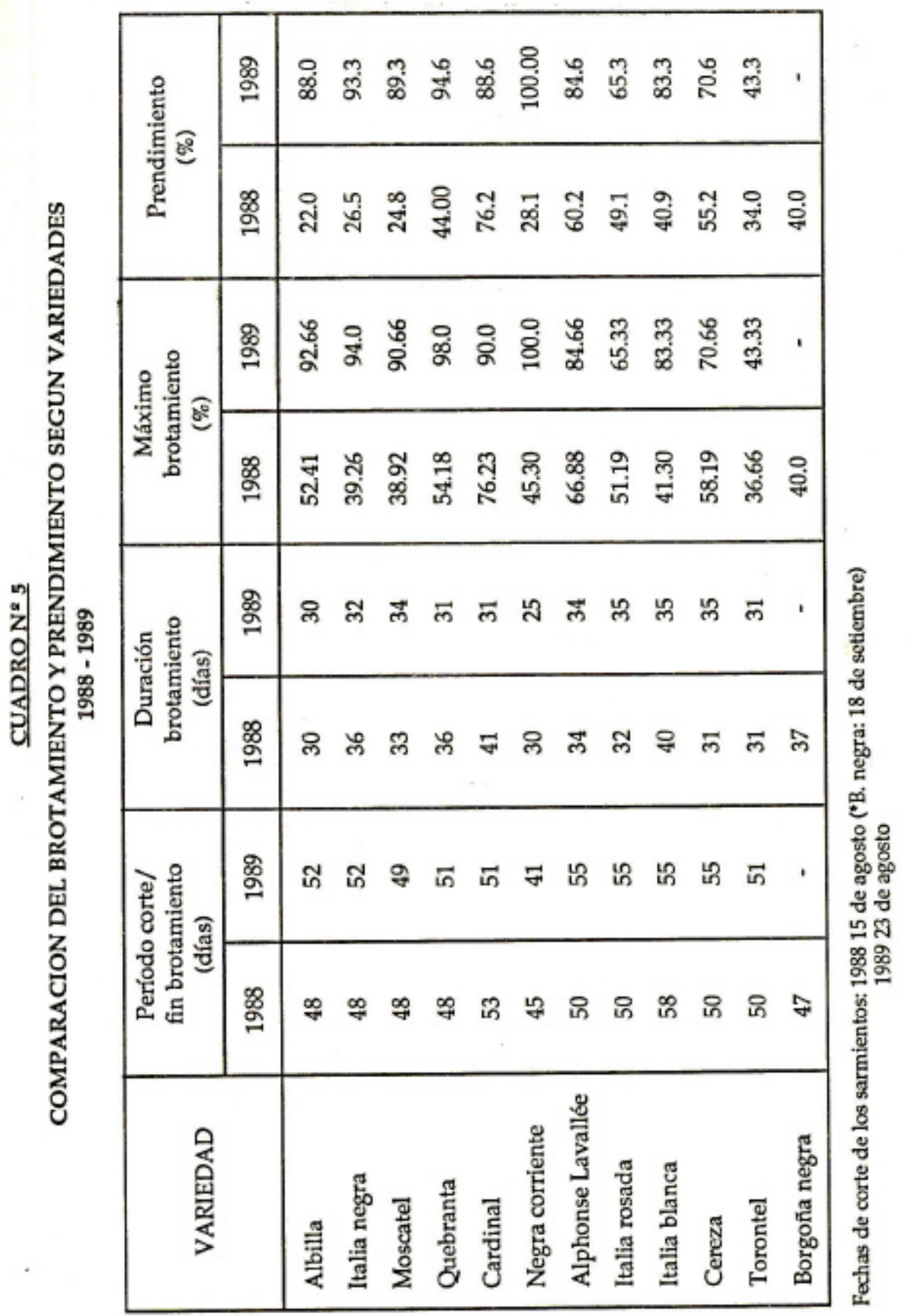


entre las 12 variedades en ensayo $(0=8.78)$, mientras que en 1989 , la diferencia entre las 11 variedades trabajadas fue notoriamente menor $(0=1.52)$. La causa fundamental se atribuye al mejoramiento tecnológico, al haberse utilizado en el último año estacas medianas y con una o dos yemas vistas, cuyo prendimiento (Cuadro $\mathrm{N} N 6$ ), fue superior que en las estacas con 3 y 4 yemas al aire. Una razón adicional para el mayor prendimiento de 1989 podría ser una mejor condición nutricia de los sarmientos que, con buena cantidad de reservas, impiden el marchitamiento de las hojas recién brotadas, hasta que las raicillas hayan formado y puedan alimentar al vástago. Este criterio ha sido remarcado en la Guía Técnica del Viticultor (4), puntualizándose que los sarmiento deben proceder de plantas productivas, vigorosas, sanas y bien agostadas, a fin de aumentar el porcentaje de brotación en el vivero. Lamentablemente, por razones administrativas, no se trabajó con las mismas plantas donadoras de sarmientos en los años estudiados. El período de brotamiento varió según los cultivares en ambos años examinados. En 1988, entre 30 y 41 días $(x=34.25 ; 0=3.60)$, y en 1989, entre $25 y 35(x=32.36 ; 0=2.80)$. La comparación de los dos años no indica correlación entre el período de brotamiento y el porcentaje de prendimiento (1988 $r=0.47 ; 1989 r$ $=0.36$ ). En los Cuadros $N 06$ y 7 se presenta el resultado de comparar el prendimiento en función del grosor de las estacas. R eafirmando las evidencias establecidas para otras latitudes, las estacas gruesas plantadas con una o dos yemas vistas prendieron en mayor proporción que las medianas y éstas que las del gadas. Teniendo en cuenta que en I ca los sarmientos del año son generalmente de mediano grosor, en 1989 se uniformizó la selección de sarmientos para el ensayo, empleando sólo estacas medianas, a I y 2 yemas; con ello, se logró un incremento de $96 \%$ en el prendimiento. En San M artín, donde la variedad de cultivo tradicional es el Borgoña, casi todas las estacas son del gadas, debido al corto período de agostamiento, por lo que la tasa de prendimiento en la zona es también menor.

En las estacas medianas, las plantadas con una sola yema al aire mostraron el mayor prendimiento, significativamente mayor que aquellas con dos yemas, lo cual debe servir de referencia para una mejor propagación en la zona.

El vigor mostrado por las variedades en la prueba de 1988 (Cuadro № 8), corresponde directamente al prendimiento $(r=0.57)$, necesitándose nuevos ensayos con menos variables para conjeturar en forma más precisa sobre esta aparente correlación. Es oportuno recordar que el vigor está influido directamente por la condición nutricia de los sarmientos, por lo que es razonable su conexión con el prendimiento.

En suma, de acuerdo a los resultados del ensayo del segundo año con estacas de grosor mediano y con 1 a 2 yemas vistas, se aprecia que la propagación de vid en las condiciones climáticas de San M artín, si es realizada mediante almácigos embolsados, economizaría un año, que es el tiempo empleado para producir barbados y evitaría los riesgos y el bajo rendimiento que señala Winkler y otros autores, cuando se planta las estacas directamente en el viñedo. Además, se obviaría las dificultades de hacer almácigos en el suelo, en las condiciones de crecimiento exuberante de maleza, propias de la zona. 


\section{CUADRO № 6}

REGISTRO NUMERICO DEL PRENDIMIENTO POR VARIEDADES SEGÚN EL GROSOR DE LASESTACAS Y CANTIDADES DE YEMAS AL AIRE 1988

\begin{tabular}{|c|c|c|c|c|c|c|c|c|}
\hline \multirow{2}{*}{ VARIEDAD } & \multirow{2}{*}{\begin{tabular}{|c} 
Pren- \\
dimiento
\end{tabular}} & \multirow{2}{*}{$\begin{array}{c}\text { Delga- } \\
\text { das }\end{array}$} & \multicolumn{4}{|c|}{ Medianas } & \multirow{2}{*}{$\begin{array}{c}\text { Grue- } \\
\text { sas }\end{array}$} & \multirow{2}{*}{ Total } \\
\hline & & & $\mathrm{M}_{1}$ & $\mathrm{M}_{2}$ & $\mathrm{M}_{3}$ & $\mathrm{M}_{4}$ & & \\
\hline Albilla & $\begin{array}{l}\mathrm{P} \\
\mathrm{N}\end{array}$ & $\begin{array}{r}7 \\
37\end{array}$ & $\begin{array}{l}4 \\
9\end{array}$ & $\begin{array}{r}47 \\
143\end{array}$ & $\begin{array}{r}8 \\
36\end{array}$ & $\begin{array}{l}1 \\
0\end{array}$ & - & $\begin{array}{r}65 \\
225\end{array}$ \\
\hline $\begin{array}{l}\text { Italia } \\
\text { negra } \\
\text { Moscatel }\end{array}$ & $\begin{array}{l}\mathrm{P} \\
\mathrm{N} \\
\mathrm{P} \\
\mathrm{N}\end{array}$ & $\begin{array}{r}2 \\
3 \\
6 \\
18 \\
\end{array}$ & $\begin{array}{l}11 \\
12 \\
13 \\
11\end{array}$ & $\begin{array}{r}45 \\
154 \\
44 \\
124\end{array}$ & $\begin{array}{l}14 \\
44 \\
91 \\
56\end{array}$ & $\begin{array}{l}0 \\
1 \\
0 \\
5 \\
\end{array}$ & $\begin{array}{r}7 \\
5 \\
2 \\
10\end{array}$ & $\begin{array}{r}79 \\
219 \\
74 \\
224 \\
\end{array}$ \\
\hline Quebranta & $\begin{array}{l}\mathrm{P} \\
\mathrm{N}\end{array}$ & $\begin{array}{l}5 \\
7 \\
\end{array}$ & $\begin{array}{l}8 \\
8\end{array}$ & $\begin{array}{l}70 \\
98 \\
\end{array}$ & $\begin{array}{l}39 \\
40 \\
\end{array}$ & $\begin{array}{l}0 \\
1 \\
\end{array}$ & $\begin{array}{l}10 \\
13 \\
\end{array}$ & $\begin{array}{l}132 \\
167 \\
\end{array}$ \\
\hline Cardinal & $\begin{array}{l}\mathrm{P} \\
\mathrm{N}\end{array}$ & $\begin{array}{l}1 \\
0\end{array}$ & $\begin{array}{r}120 \\
28\end{array}$ & $\begin{array}{l}82 \\
37\end{array}$ & $\begin{array}{l}2 \\
1\end{array}$ & - & $\begin{array}{r}26 \\
6 \\
\end{array}$ & $\begin{array}{r}231 \\
72\end{array}$ \\
\hline $\begin{array}{l}\text { Negra } \\
\text { corrienté }\end{array}$ & $\begin{array}{l}\mathrm{P} \\
\mathrm{N}\end{array}$ & $\begin{array}{l}1 \\
4\end{array}$ & $\begin{array}{l}24 \\
45\end{array}$ & $\begin{array}{r}57 \\
150 \\
\end{array}$ & $\begin{array}{r}2 \\
17 \\
\end{array}$ & - & $\begin{array}{l}3 \\
6 \\
\end{array}$ & $\begin{array}{r}87 \\
222 \\
\end{array}$ \\
\hline $\begin{array}{l}\text { Alphonse } \\
\text { Lavallée }\end{array}$ & $\begin{array}{l}\mathrm{P} \\
\mathrm{N}\end{array}$ & $\begin{array}{l}7 \\
5 \\
\end{array}$ & $\begin{array}{l}59 \\
45\end{array}$ & $\begin{array}{r}102 \\
65\end{array}$ & $\begin{array}{l}0 \\
2\end{array}$ & - & $\begin{array}{r}12 \\
2 \\
\end{array}$ & $\begin{array}{l}180 \\
119\end{array}$ \\
\hline $\begin{array}{l}\text { Italia } \\
\text { rosada }\end{array}$ & $\begin{array}{l}\mathrm{P} \\
\mathrm{N}\end{array}$ & $\begin{array}{l}1 \\
9 \\
\end{array}$ & $\begin{array}{l}71 \\
66 \\
\end{array}$ & $\begin{array}{l}70 \\
73 \\
\end{array}$ & $\begin{array}{l}1 \\
0\end{array}$ & - & $\begin{array}{l}1 \\
1 \\
\end{array}$ & $\begin{array}{l}144 \\
149 \\
\end{array}$ \\
\hline $\begin{array}{l}\text { Italia } \\
\text { blanca }\end{array}$ & $\begin{array}{l}\mathrm{P} \\
\mathrm{N}\end{array}$ & $\begin{array}{l}0 \\
5\end{array}$ & $\begin{array}{l}58 \\
59\end{array}$ & $\begin{array}{l}36 \\
91 \\
\end{array}$ & $\begin{array}{l}1 \\
1 \\
\end{array}$ & - & $\begin{array}{r}18 \\
7\end{array}$ & $\begin{array}{l}113 \\
163 \\
\end{array}$ \\
\hline Cereza & $\begin{array}{l}\mathrm{P} \\
\mathrm{N}\end{array}$ & $\begin{array}{l}0 \\
1 \\
\end{array}$ & $\begin{array}{l}64 \\
63 \\
\end{array}$ & $\begin{array}{l}92 \\
68 \\
\end{array}$ & - & - & $\begin{array}{l}9 \\
2 \\
\end{array}$ & $\begin{array}{l}165 \\
134 \\
\end{array}$ \\
\hline Torontel & $\begin{array}{l}\mathrm{P} \\
\mathrm{N}\end{array}$ & $\begin{array}{l}0 \\
6 \\
\end{array}$ & $\begin{array}{r}63 \\
112 \\
\end{array}$ & $\begin{array}{l}32 \\
76 \\
\end{array}$ & - & - & $\begin{array}{l}7 \\
4 \\
\end{array}$ & $\begin{array}{l}102 \\
198 \\
\end{array}$ \\
\hline $\begin{array}{l}\text { Borgoña } \\
\text { negra }\end{array}$ & $\begin{array}{l}\mathrm{P} \\
\mathrm{N}\end{array}$ & - & - & $\begin{array}{l}62 \\
93 \\
\end{array}$ & - & - & - & $\begin{array}{l}62 \\
93 \\
\end{array}$ \\
\hline TOTAL & $\begin{array}{l}\mathrm{P} \\
\mathrm{N}\end{array}$ & $\begin{array}{l}28 \\
95 \\
\end{array}$ & $\begin{array}{l}495 \\
458 \\
\end{array}$ & $\begin{array}{c}739 \\
1172 \\
\end{array}$ & $\begin{array}{r}76 \\
197 \\
\end{array}$ & $\begin{array}{l}1 \\
7 \\
\end{array}$ & $\begin{array}{l}95 \\
56 \\
\end{array}$ & $\begin{array}{l}1434 \\
1978\end{array}$ \\
\hline Porcentaje de Prer & miento & 22.7 & 51.9 & 38.6 & 27.8 & 12.5 & 62.9 & 42.02 \\
\hline
\end{tabular}




\section{CUADRO № 7}

REGISTRO PORCENTUAL DEL RENDIMIENTO POR VARIEDADES SEGÚN EL GROSOR DE LA ESTACAS Y CANTIDAD DE YEMAS AL AIRE-1989

\begin{tabular}{|c|c|c|c|c|c|c|}
\hline \multirow[b]{2}{*}{ VARIEDAD } & \multirow[b]{2}{*}{$\begin{array}{c}\text { Delgad } \\
\text { as }\end{array}$} & \multicolumn{3}{|c|}{ Medianas } & \multirow{2}{*}{$\begin{array}{c}\text { Gruesas } \\
(*)\end{array}$} & \multirow{2}{*}{$\begin{array}{c}\text { Prendimiento } \\
\text { Total }\end{array}$} \\
\hline & & $M_{1}$ & $M_{2}$ & $M_{3}$ & & \\
\hline Albilla & 12 & 30 & 25 & 18 & - & 22.0 \\
\hline Italia negra & $\mathbf{N} / \mathbf{S}$ & 48 & 23 & 24 & 58 & 26.5 \\
\hline M oscatel & 25 & 54 & 26 & 14 & 17 & 24.8 \\
\hline Quebranta & 42 & 50 & 42 & 50 & 43 & 44.0 \\
\hline Cardinal & $\mathbf{N} / \mathbf{S}$ & 81 & 69 & $\mathbf{N} / \mathbf{S}$ & 81 & 76.2 \\
\hline Negra corriente & $\mathbf{N} / \mathbf{S}$ & 35 & 27 & 10 & $\mathbf{N} / \mathbf{S}$ & 28.1 \\
\hline Alphonse L avalleé & 58 & 57 & 61 & $\mathbf{N} / \mathbf{S}$ & 86 & 60.2 \\
\hline Italia Rosada & 10 & 52 & 49 & $\mathbf{N} / \mathbf{S}$ & $\mathbf{N} / \mathbf{S}$ & 49.1 \\
\hline Italia blanca & $\mathbf{N} / \mathbf{S}$ & 50 & 28 & $\mathbf{N} / \mathbf{S}$ & 72 & 40.9 \\
\hline Cereza & $\mathbf{N} / \mathbf{S}$ & 50 & 57 & - & 82 & 55.2 \\
\hline Torontel & N/S & 36 & 29 & - & 64 & 34.0 \\
\hline Borgoña negra & - & - & 40 & - & - & 40.0 \\
\hline
\end{tabular}

N/S = Muestra no significativa $\quad M n=E I$ subíndice señala el número de yemas al aire

(*) De las 151 estacas gruesas, 83 (54\%) se plantaron con 1 yema al aire, 61 (40\%) con 22 yemas y sólo $7(4 \%)$ con 3 yemas al aire.

CUADRO № 8

COMPARACION DEL VIGOR CON EL PRENDIMIENTO -1988

\begin{tabular}{|l|c|c|}
\hline \multicolumn{1}{|c|}{ VARIEDAD } & Prendimiento & $\begin{array}{l}\text { V igor } \\
\text { (C m) }\end{array}$ \\
\hline Cardinal & & 24.20 \\
Alphonse Lavalleé & 76.2 & 26.50 \\
Cereza & 60.2 & 14.82 \\
Italia R osada & 55.2 & 17.63 \\
Quebranta & 49.1 & 25.03 \\
Italia blanca & 44.0 & 16.97 \\
Borgoña negra (T) & 40.9 & 15.66 \\
Torontel & 40.0 & 15.25 \\
Negra corriente & 34.0 & 12.42 \\
Italia negra & 28.1 & 15.66 \\
M oscatel & 26.5 & 19.63 \\
Albilla & 24.8 & 17.45 \\
\hline
\end{tabular}




\section{CONCLUSIONES}

1. La temperatura, con variaciones diarias entre 18 y 34 grados centígrados, acelera el proceso de prendimiento de las estacas en el almácigo embolsado.

2. L a tierra arenosa es un buen sustrato para almácigos de vid embolsados.

3. Sometidos a iguales condiciones climáticas y agronómicas los cultivos estudiados exhiben diferentes grados de prendimento.

4. Las estacas, con madera de 1 año, varían su capacidad de prendimiento cn función directa a su diámetro e inversa al número de yemas vistas.

5. Los cultivares estudiados mostraron un período promedio de brotamiento de 34 y32 días en los años 1988 y 1989, respectivamente.

6. No existe correlación entre el grado de prendimiento con el período de prebrotamiento, ni con el lapso de brotamiento entre los dos años (1988 y 1989).

7. La propagación de vid en almácigo embolsado empleando estacas sin injertar medianas o gruesas con una o dos yemas vistas ha mostrado un alto grado de prendimiento. Además, facilita el control de humedad del sustrato, el manejo fitosanitario de las estacas en brote y el transporte y plantado de los vástagos.

8. Las bolsas de polietileno perforadas, de $30 \times 15 \mathrm{~cm}$. de tamaño, han demostrado funcionalidad para constituir almácigos de vid.

9. Es necesario repetir dos veces más el ensayo descrito, controlando la identidad y estado de las plantas donantes, para determinar con mayor precisión la aptitud de los cultivares en este tipo de propagación.

10. Las variedades que se han comportado mejor durante los años 1988 y 1989, en relación con el brotamiento y prendimiento, fueron la Cardinal y la Alphonse L avallée.

\section{BIBLIOGRAFIA}

GARAYAR, H.A. 1987. Diagnóstico de la Zona Vitivinícola de San Antonio de Cumbaza, Tarapoto, INIAA, Informe de viaje. 4 pág.

LARREA, A 1981. Viticultura Básica, Barcelona, AED OS, 267 pág.

MADERO, E. et al 1977. Comportamiento y A daptación de cultivares de Vid libres de virus bajo las condiciones ecológicas de la Comarca Lagunera. México. Viticultura CIAN 77 pp. 180-238

MATAM OROS; Coah. Campo Agrícola Experimental La Laguna 1984. Guía Técnica del Viticultor. México. Secretaría de Agricultura y Recursos Hidráulicos. Instituto Nacional de Investigaciones A grícolas del Norte. 245 pág.

RODRIGUEZ, R. y Ruesta, A. 1982. Cultivo de la vid en el Perú. Serie Manual Técnico N 2 2. M inisterio de A gricultura, INIPA 174 pág.

WINKLER, A. J. et al 1984. General Viticulture. Berkely Ca. EUA, University California Press. 710 pág. 